\title{
Thermodynamics and feature extraction by machine learning
}

\author{
Shotaro Shiba Funai $\odot^{1, *}$ and Dimitrios Giataganas $\circledast^{2, \dagger}$ \\ ${ }^{1}$ Physics and Biology Unit, Okinawa Institute of Science and Technology (OIST), 1919-1 Tancha, Onna-son, \\ Kunigami-gun, Okinawa 904-0495, Japan \\ ${ }^{2}$ Physics Division, National Center for Theoretical Sciences, National Tsing-Hua University, Hsinchu 30013, Taiwan
}

(Received 23 December 2019; accepted 26 August 2020; published 15 September 2020)

\begin{abstract}
Machine learning methods are powerful in distinguishing different phases of matter in an automated way and provide a new perspective on the study of physical phenomena. We train a restricted Boltzmann machine (RBM) on data constructed with spin configurations sampled from the Ising Hamiltonian at different values of temperature and external magnetic field using Monte Carlo methods. From the trained machine we obtain the flow of iterative reconstruction of spin state configurations to faithfully reproduce the observables of the physical system. We find that the flow of the trained RBM approaches the spin configurations of the maximal possible specific heat which resemble the near-criticality region of the Ising model. In the special case of the vanishing magnetic field the trained RBM converges to the critical point of the renormalization group (RG) flow of the lattice model. Our results suggest an explanation of how the machine identifies the physical phase transitions, by recognizing certain properties of the configuration like the maximization of the specific heat, instead of associating directly the recognition procedure with the RG flow and its fixed points. Then from the reconstructed data we deduce the critical exponent associated with the magnetization to find satisfactory agreement with the actual physical value. We assume no prior knowledge about the criticality of the system and its Hamiltonian.
\end{abstract}

DOI: 10.1103/PhysRevResearch.2.033415

\section{INTRODUCTION}

Machine learning (ML) has been recently used as a very effective tool for the study and prediction of data in various fields of physics. Due to the combination of availability of large amounts of data and the advances in computational power, it has become a powerful method [1,2]. Deep learning techniques have yielded with striking efficiency results on a diverse set of complicated ML tasks in computer vision, speech recognition, natural language processing, and more recently, physics. However, despite such enormous success, relatively little is understood theoretically about why these techniques are so successful.

The recent applications of ML on physics provide a solid hope that a more fundamental link between the methods of learning and certain physical models could established that will be mutually beneficial for understanding deeper certain aspects in both sciences. The ML on physical models is performed with neural networks (NNs), composed of nodes and edges. These are of two major types, the supervised learning where the network is trained on data with certain labeling, and perhaps a more effective way of unsupervised learning,

\footnotetext{
*shotaro.funai@oist.jp

${ }^{\dagger}$ dimitrios.giataganas@cts.nthu.edu.tw
}

Published by the American Physical Society under the terms of the Creative Commons Attribution 4.0 International license. Further distribution of this work must maintain attribution to the author(s) and the published article's title, journal citation, and DOI. where the strategy is to learn the full distribution of the data and no labels are required. Both approaches have been applied on physical models and particularly on the Ising model, where unsupervised methods have been used extensively to identify phase transitions and order parameters of the Ising model using images of classical spin configurations [3-6], while some parallel work with supervised methods includes Refs. [7-9]. Such methods have been proven useful and strikingly successful for the study of several modern complex physical problems, like the strongly correlated many-body systems [10], overcoming the challenges originating from the difficulty of describing the nontrivial correlations encoded in the exponential complexity of the many-body wave function [11-14], in the search for the exotic particles [15], in the study of the liquid-glass phase transition [16], and in identifying phase transitions by circumventing the fermion sign problem [17]. Such applications of ML in identifying and classifying different phases of matter, including the topological ones, include Refs. [18-23]. Moreover, attempts to understand the mechanism of the emergence of bulk geometries in the gauge-gravity correspondence have been also proposed in Refs. [24,25]. Most of these physical applications are summarized in an analytic review, Ref. [26].

As part of this revolution, a more fundamental relation has been proposed between the way that the learning occurs in neural networks and the renormalization group (RG) flow in statistical Ising models [27-33]. In [33] particularly, a deeper relation has been demonstrated between the two ideas, where a learning algorithm has identified the relevant degrees of freedom of the Ising model without any prior knowledge about the system. The connection between RG and ML lies in the idea 
behind the RG flow, where by identifying relevant degrees of freedom and integrating out the irrelevant ones iteratively, one arrives at a low-energy effective theory with universal features. For example, in the block spin decimation procedure the spins are grouped into blocks coupled to their nearest neighbors, and then treated again as random variables. This kind of locality is exploited by artificial systems, where the first neural layer performs mostly only the local operations. In analogy to the RG flow, by adding layers to the network, the correlation between hidden variables is expected to decrease as we enter deeper into the network.

Furthermore, in [34] it has been shown that the critical fixed point of the RG flow and the fixed point of data reconstruction by the ML techniques are coincident. The restricted Boltzmann machine (RBM) [35] plays a fundamental role in this task. It is a stochastic neural undirected network, where its neuron has a binary value that has a probabilistic element depending on its neighboring units, resembling vaguely properties of the Ising model. The RBM has been used for learning the configurations of the Ising model in the absence of external fields and then reconstructing these configurations iteratively. We will be using such methods of learning in our work and we will call the resulting flow of such series of iteratively reconstructed configurations an "RBM flow."

One motivation of our work is to study whether and when the RBM flow is correlated with the RG flow and when it tends to the critical fixed point of the RG flow. In our analysis we generate RBM flows, in systems such that when certain parameters are fixed, the critical fixed points of the RG flow can be inaccessible, while still the noncritical fixed points remain accessible. For this purpose we choose to work with the one-dimensional and the two-dimensional Ising models in the presence of external magnetic field. Focusing mostly on the two-dimensional model, we demonstrate that for fixed nonvanishing magnetic field, the RBM reconstruction data flows to configurations that resemble closer a configuration of the phase transition. It recognizes as "critical" points the ones which are close to maximizing the specific heat for given external parameters. The RBM flow does not seem to show any strong tendency to approach the noncritical fixed points of the RG flow. At the special case of zero magnetic field where an unstable RG critical point exists at a finite temperature, we confirm that the RBM reconstruction data flow to it and recognize it. Our analysis yields an alternative explanation on the way that the RBM identifies the phase transitions, which is not necessarily related to the RG flow.

Having studied the properties of the RBM flow and how it recognizes the phase transitions, the second motivation of our paper is to demonstrate the computation of the thermodynamic quantities on the samples generated by the Boltzmann machines to show that they closely reproduce those calculated directly from the Monte Carlo samples. For the zero magnetic field model and without giving information to the machine about the Hamiltonian of the system and its criticality, we compute the critical exponent associated with magnetization from the reconstructed data to find satisfactory agreement.

We emphasize that the training and reconstruction process is based entirely on the spin configurations without any additional input. The RBM is trained by a large number of Monte Carlo (MC) spin microstate configurations in various temperatures. The binary MC configurations can be thought as being transformed to 1- or 2-dimensional images, depending on the dimension of the Ising model, with binary pixel values corresponding to the up and down spins. This is all the knowledge that the RBM has access to for the physical system; it has no further information for the existence of the critical points and their properties. During the unsupervised training the RBM extracts specific patterns through the KullbackLeibler (KL) divergence minimization based on the images we provide. Then to define the RBM flow we choose MC spin configurations at a particular temperature and magnetic field away from the criticality and we ask the RBM to reconstruct them, i.e., to produce a new spin microstate according to its prior training. The outcome of the new spin configuration corresponds in principle to a different temperature and magnetic field. By repeating this process on the produced configuration, we construct the RBM flow which has the physical properties we describe in our work.

We organize our paper as follows. In Sec. II we present the properties of the Ising model and the RG flow in $1 \mathrm{~d}$ and $2 \mathrm{~d}$ lattices, which will be useful in the next sections. In Sec. III we explain our method of ML to learn the spin configurations in the Ising model. In Sec. IV we present the results of the RBM flows. Then we demonstrate how to evaluate the critical exponent of the Ising model using the RBM configuration in Sec. V, and we finish by discussing our results in Sec. VI.

\section{THE ISING MODEL}

In this section we briefly establish the setup and review aspects of the thermodynamics and the renormalization group flow of the Ising model on $1 \mathrm{~d}$ and $2 \mathrm{~d}$ lattices. The Ising model is built on a lattice of $N$ sites. Each site, labeled by the index $i$, contains a spin $s_{i}$ with values \pm 1 which represent the two possible states. The Hamiltonian is

$$
\mathcal{H}=-J \sum_{\langle i j\rangle} s_{i} s_{j}-H \sum_{i} s_{i},
$$

where $\langle i j\rangle$ denotes all the nearest neighbor pairs of the sites $i$ and $j, J>0$ is the coupling of the nearest neighbors, and $H$ is the external magnetic field. The magnetization $M$ of this system is defined as the sum of all the spins $M=\sum_{i} s_{i}$.

The partition function of the system reads

$$
\mathcal{Z}=\sum_{\{s\}} \prod_{i} e^{K s_{i} s_{i+1}+h s_{i}},
$$

where $K:=J / T$ and $h:=H / T$, while the Boltzmann constant is set equal to unit, $k_{B}=1$. In practice the lattice has a finite number of spins and the boundary conditions affect the system's properties. In the finite- $N$ spin chain two boundary conditions may be applied, the toroidal boundary condition where the last $N$ th spin interacts with the first spin forming a ring, where all the spins are equivalent; or the free-end boundary condition where the first and last spin have only one neighbor. Nevertheless, by considering the thermodynamic limit $N \rightarrow \infty$ the effect of the boundary conditions becomes negligible. 


\section{A. Exact solution of 1d Ising model}

By adopting the toroidal boundary conditions $s_{N+1}=s_{1}$, where all spins are equivalent, the energy of the 1d Ising model takes the form

$$
\frac{E}{T}=-K \sum_{i=1}^{N} s_{i} s_{i+1}-\frac{h}{2} \sum_{i=1}^{N}\left(s_{i}+s_{i+1}\right) .
$$

The partition function of the system (2) can be written in terms of the elements of the transfer matrix $\mathbf{T}$ as

$$
\mathcal{Z}=\sum_{\{s\}} \prod_{i=1}^{N} T_{s_{i} s_{i+1}},
$$

where the symmetric $2 \times 2$ matrix

$$
T_{s_{i} s_{i+1}}:=\exp \left[K s_{i} s_{i+1}+\frac{h}{2}\left(s_{i}+s_{i+1}\right)\right]
$$

has the diagonal elements $\left(T_{++}, T_{--}\right)$and the nondiagonal ones $T_{+-}=T_{-+}$. The matrix $T$ has the property $\left(\mathbf{T}^{2}\right)_{s_{1} s_{3}}=$ $\sum_{s_{2}} T_{s_{1} s_{2}} T_{s_{2} s_{3}}$ which leads to the simplification of the partition function as $Z_{N}=\operatorname{Tr}\left(\mathbf{T}^{N}\right)$. By using a similarity transformation on $\mathbf{T}$, we diagonalize it to get a matrix $\mathbf{T}^{\prime}=\operatorname{diag}\left(\lambda_{+}, \lambda_{-}\right)$, where $\lambda_{ \pm}$are the eigenvalues of $\mathbf{T}$,

$$
\lambda_{ \pm}=e^{K} \cosh h \pm\left(e^{-2 K}+e^{2 K} \sinh ^{2} h\right)^{1 / 2} .
$$

The partition function becomes $Z_{N}=\lambda_{+}^{N}+\lambda_{-}^{N}$, which in the thermodynamic limit simplifies further to $Z_{N}=\lambda_{+}^{N}$.

The magnetization of the system is defined as $M=$ $-(\partial F / \partial H)_{T}$, where the free energy is given by $F=-T \ln Z$ leading to the magnetization per site

$$
m:=\frac{M}{N}=\frac{\sinh h}{\sqrt{\sinh ^{2} h+e^{-4 K}}} .
$$

Therefore, the $1 \mathrm{~d}$ Ising model becomes a ferromagnet only at $T=0$, where $m \rightarrow 1$. The specific heat of the system is obtained by $C=(\partial E / \partial T)_{H}$, where the energy is be given by $E=T^{2}(\partial \log Z / \partial T)_{H}$.

\section{B. Analytic solutions of $2 \mathrm{~d}$ Ising model}

The $2 \mathrm{~d}$ Ising model has been solved exactly for zero magnetic field in [36] and studied further in [37]. The critical temperature at the second-order phase transition is given by $T_{c} / J=2 / \log (1+\sqrt{2}) \simeq 2.27$, and the magnetization for $T<T_{c}$ is given by

$$
m=\left(1-\sinh ^{-4} 2 K\right)^{1 / 8} .
$$

For general $T$, the specific heat is a given by a more involved formula

$$
\begin{aligned}
C= & \frac{1}{\pi}(K \operatorname{coth} 2 K)^{2}\left\{K_{1}(\kappa)-E_{1}(\kappa)\right. \\
& \left.-\frac{1}{\cosh ^{2} 2 K}\left[\frac{\pi}{2}+\left(2 \tanh ^{2} 2 K-1\right) K_{1}(\kappa)\right]\right\},
\end{aligned}
$$

where $K_{1}(\kappa)$ and $E_{1}(\kappa)$ are the complete elliptic integrals of the first and second kind, respectively, and $\kappa:=$ $2 \sinh 2 K / \cosh ^{2} 2 K$. The specific heat diverges at $T=$ $T_{c}, H=0$, signaling the phase transition of the system.
The exact solution with nonvanishing magnetic field is not known although powerful approximation techniques are applicable. In the Bethe-Peierls approximation we consider a central spin $s_{0}$ and the exact interaction with its nearest neighbors $s_{1}, \ldots, s_{q}$, while the rest of the spins are approximated to act on the nearest neighbors through a self-consistent effective field. The energy can be written as

$$
\frac{E}{T}=-\left(K s_{0}+h_{\mathrm{eff}}\right) \sum_{j=1}^{q} s_{j}-h s_{0},
$$

where $q$ is the number of nearest spins and $h_{\mathrm{eff}}=h+(q-$ 1) $m / T$ is the effective field which takes into account contributions of the external magnetic field $h$ and the magnetization $m$ from the rest of the spins. The translational invariance of the model implies that $\left\langle s_{0}\right\rangle=\left\langle s_{i}\right\rangle$, resulting in the equation for the effective field

$$
\cosh ^{q-1}\left(K+h_{\mathrm{eff}}\right)=e^{2\left(h_{\mathrm{eff}}-h\right)} \cosh ^{q-1}\left(K-h_{\mathrm{eff}}\right),
$$

which when solved gives the magnetization $m$ of the system. In the $2 \mathrm{~d}$ Ising model with the square lattice where $q=4$, the approximate rapid decrease of the magnetization happens at $T / J=2.89, H=0$, giving a prediction of the critical temperature, close enough to the exact value $T_{c} / J=2.27$.

\section{Renormalization group of Ising model}

By applying RG flow methods the fixed and critical points of the system are identified. The idea of renormalization in the Ising model is to transform the original Hamiltonian $\mathcal{H}$ into a deformed Hamiltonian $\mathcal{H}^{\prime}=\mathcal{R} \mathcal{H}$ where an operation $\mathcal{R}$ removes a number of degrees of freedom. The degrees of freedom are reduced as $N^{\prime}=b^{-d} N$, where $N$ and $N^{\prime}$ are the original and transformed number of lattice sites, $d$ is the dimensionality of the lattice, and $b$ is a constant which denotes how the decimation is performed.

This transformation is realized by carrying out a partial trace to obtain a new partition function that has fewer summations on the remaining sites. The resulting Hamiltonian $\mathcal{H}^{\prime}$ should be algebraically specified to take the same functional form as the original Hamiltonian with rescaled coefficients and most likely new interactions. However, the partition function remains proportional to the original one $Z_{N^{\prime}}\left(\mathcal{H}^{\prime}\right)=c Z_{N}(\mathcal{H})$, where $c$ depends on the couplings of the theory. Therefore, the free energy remains the same, while the free energy per site in the new Hamiltonian will increase by a rescaling $b^{d}$, resulting in the length of lattice spacing being rescaled by $b^{-1}$ and respectively momenta being rescaled by $b$.

The fixed points in this procedure which interest us in this work are defined by the equality $\mathcal{H}^{\prime}=\mathcal{H}$. At such points, the system is invariant under scale changes; thus the correlation length is invariant. In particular, critical fixed points where the phase transition of the system happens have infinite correlation lengths. 


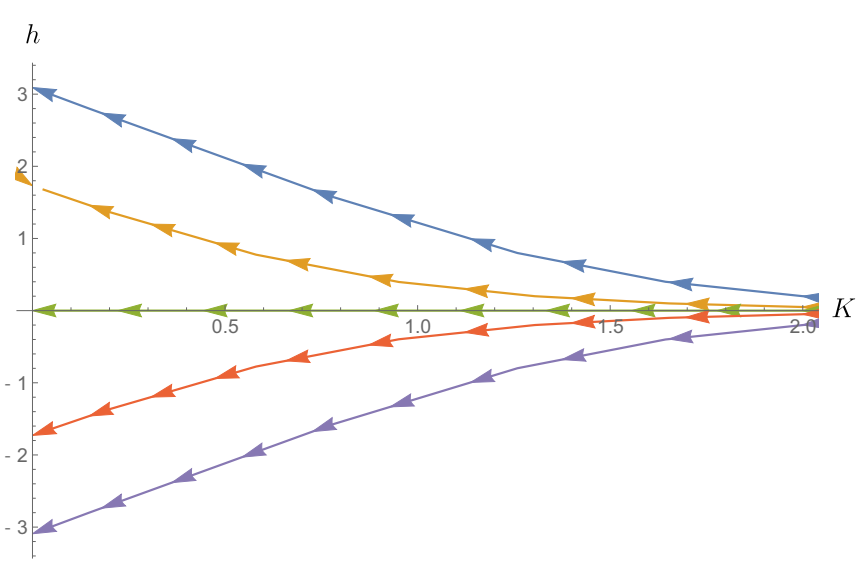

FIG. 1. The RG flow in the 1d Ising model with external magnetic field. The region $K \rightarrow 0$ represents the high-temperature regime, while $K \rightarrow \infty$ represents the low-temperature regime.

\section{RG flow in 1d Ising model}

By implementing the decimation procedure of summing over the sites with even index $i$ we have

$$
Z(N, K, h)=e^{g N} Z\left(\frac{N}{2}, K^{\prime}, h^{\prime}\right),
$$

where $g$ depends on the coupling constants $K, h$, and the rescaled Hamiltonian $\mathcal{H}^{\prime}$ is a coarse-grained version of the original one with half degrees of freedom and primed couplings. The recursive equation (12) given a system $K^{\prime}(K, h), h^{\prime}(k, h), g(K, h)$ generates the diagram of Fig. 1. In the process we have removed half of the degrees of freedom $N^{\prime}=N / 2$ and at the same time we have doubled the lattice spacing $\alpha^{\prime}=2 \alpha$, while the correlation length has been decreased to half of the original one. The renormalization of the $1 \mathrm{~d}$ Ising model is exact; that is, the coarse-grained Hamiltonian does not have additional terms which could correspond to new interactions between the spins. The number of the new coupling constants remains the same as the couplings before the decimation, which are expressed exclusively in terms of the initial ones.

The fixed points where our couplings $K$ and $h$ do not change under successive decimation are (i) at the infinite temperature where the spins are completely disordered and the correlation length tends to be zero; (ii) at zero temperature and magnetic field, which is the critical unstable point, where the correlation length diverges; (iii) at zero temperature and infinite magnetic field where the spins are completely ordered. The RG flow of the 1d Ising model is depicted in Fig. 1.

\section{E. RG flow in 2d Ising model}

As the 1d Ising model, we take the partial trace over the nearest and next-nearest neighbors, where the procedure in each row and column of the $2 \mathrm{~d}$ lattice resembles the decimation in the $1 \mathrm{~d}$ spin chain. The coarse-grained lattice comes by tracing out the center spin of each cross interaction among spins, which leads to a lengthy expression of the partition function, written in a compact form as

$$
Z(N, K, h)=e^{N^{\prime} g} Z\left(N^{\prime}, K_{i}^{\prime}, h^{\prime}\right) .
$$

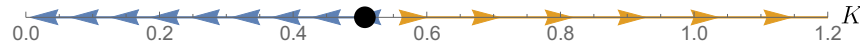

FIG. 2. The RG flow of the $2 \mathrm{~d}$ Ising model without a magnetic field $h=0$, when the square interactions $K_{3}^{\prime}$ are negligible (13). The fixed point $K_{c}=J / T_{c}=0.507$ is depicted and is unstable.

The renormalized Hamiltonian $\mathcal{H}^{\prime}$ is the Landau-GinzburgWilson energy function with three couplings. $K_{1}^{\prime}$ is the renormalized coupling between nearest neighbors, $K_{2}^{\prime}$ is the new interaction coupling between next-nearest neighbors, and $K_{3}^{\prime}$ is the four-spin interaction of any square in the lattice. To solve this recursive equation we need to consider the four independent spin configurations around a central spin, which give analytical relations of the coupling constants $K_{i}$. Subsequent decimation leads to more involved interactions each time, and an approximation needs to be considered in order to find the fixed point analytically. By assuming that the nearest and next-nearest neighbor interactions dominate, we use the perturbation expansion, to get from the iteration equation (13),

$$
K^{\prime} \equiv K_{1}^{\prime}+K_{2}^{\prime}=\frac{3}{8} \log \cosh 4 K .
$$

The fixed points are at $K=0, \infty$ and $K=K_{c}=0.507$. The critical point is unstable and, as depicted in Fig. 2, the flow is either toward the high-temperature paramagnetic fixed point $K=0$ or toward the low-temperature ferromagnetic fixed point $K=\infty$. Note that the value of $K_{c}=J / T_{c}$ is slightly different from the exact value $T_{c}=2.27$ due to the approximation applied during the decimation.

The above analysis can be repeated for the case of positive magnetic field $H$, where there are no critical points except the critical unstable point $T=T_{c}, H=0$ as shown above.

\section{MACHINE LEARNING}

\section{A. Unsupervised learning by restricted Boltzmann machines}

Restricted Boltzmann machines (RBMs) are generative stochastic models commonly used in unsupervised learning. The RBM parameters contain a set of weights and biases corresponding to the couplings and local fields present in the system. We construct the energy as a function of the data input, which can be expressed as a Gibbs-Boltzmann probability density for the binary and shallow RBM with one hidden layer

$$
p\left(v_{i}, h_{a}\right)=\frac{e^{-\Phi\left(v_{i}, h_{a}\right)}}{\mathcal{Z}},
$$

where $\Phi$ is the "energy" function

$$
\Phi\left(v_{i}, h_{a}\right)=-\sum_{i, a} v_{i} W_{i a} h_{a}-\sum_{i} b_{i}^{(v)} v_{i}-\sum_{a} b_{a}^{(h)} h_{a},
$$

$W_{i a}$ is the weight matrix, and $\mathcal{Z}$ is the partition function $\mathcal{Z}=$ $\sum_{\left\{v_{i}, h_{a}\right\}} e^{-\Phi\left(v_{i}, h_{a}\right)}$. The set $\left(b_{i}^{(v)}, b_{a}^{(h)}\right)$ can be regarded as the local potentials defining the biases on each variable assigned in the visible and hidden neurons $\left(v_{i}, h_{a}\right)$, respectively.

An exact computation of the partition function is in practice inaccessible; however a precise estimate of the normalization is unnecessary for the RBM application in the Ising model. The RBM bipartite structure admits couplings between the hidden and visible variables, which can be effectively used 
to construct efficient sampling schemes by the use of the contrastive divergence (CD) method [38]. The method is based of a sampling Markov chain alternating between samples drawn from the conditional probabilities of each layer which depend on the conditional expectations of the previously sampled layer.

To train the parameters of the RBM for our data set, we minimize the distance between the probability distributions of the input data $q\left(v_{i}\right)$ and the output data $p\left(v_{i}\right)$. The input here is the training data of the Ising model for a set of values $(T, H)$ and the output is what the RBM reconstructs. The distance is measured using the $\mathrm{KL}$ divergence $\mathrm{KL}(q \| p)$, and we successively decrease it by renewing the weight matrix and biases, as $W_{i a} \rightarrow W_{i a}-\epsilon \frac{\partial \mathrm{KL}(q \| p)}{\partial W_{i a}}$. We point out that the learning method of reweighting that we use may be correlated with our final findings. The parameter $\epsilon$ is the learning rate which we set $10^{-3}$ to achieve an optimal ratio of performance over computational time required. The process is done by considering an average of a number of data points, where the needed gradients for the process are given by

$$
\begin{gathered}
\frac{\partial \mathrm{KL}(q \| p)}{\partial W_{i a}}=\left\langle v_{i} h_{a}\right\rangle_{p\left(h_{a} \mid v_{i}\right)}-\left\langle v_{i} h_{a}\right\rangle_{p\left(v_{i}, h_{a}\right)}, \\
\frac{\partial \mathrm{KL}(q \| p)}{\partial b_{i}^{(v)}}=\left\langle v_{i}\right\rangle_{q\left(v_{i}\right)}-\left\langle v_{i}\right\rangle_{p\left(v_{i}, h_{a}\right)}, \\
\frac{\partial \mathrm{KL}(q \| p)}{\partial b_{a}^{(h)}}=\left\langle h_{a}\right\rangle_{p\left(h_{a} \mid v_{i}\right)}-\left\langle h_{a}\right\rangle_{p\left(v_{i}, h_{a}\right)},
\end{gathered}
$$

where $\langle\mathcal{A}\rangle_{p}$ is the expectation value of $\mathcal{A}$ with respect to the probability density $p$ and $p\left(h_{a} \mid v_{i}\right)$ is the probability density of $h_{a}$ for given $v_{i}$ with the probability density $q\left(v_{i}\right)$. In our work to optimize the weight matrix and biases, we use a modification of the method [38], taking advantage of the iterative gradient behavior to quickly obtain thermalized Markov chains through Gibbs sampling at one step (CD-1) [39]. We evaluate the gradients by running the Markov chain with the probability distribution $p\left(v_{i}, h_{a}\right)$ in one step, starting from the input data $q\left(v_{i}\right)$.

The number of times of successive renewal, the so called learning epoch, is chosen to be $10^{4}$. We choose an RBM with $N_{v}=100$ neurons in the visible layer, so that we can input the spin configurations $\left\{s_{i}\right\}$ to the visible neurons $v_{i}$, and we study the cases of $N_{h}=9,16,25,36,49,64,81,100$ neurons in the hidden layer.

For making the training data set we use the method of the Metropolis MC simulation, to generate $1 \mathrm{~d}$ and $2 \mathrm{~d}$ Ising configurations $\left\{s_{i}\right\}$ at various values of temperature $T=0,0.5, \ldots, 9.5$ and external magnetic field $H=$ $0,0.5, \ldots, 4.5$. The $1 \mathrm{~d}$ chain size is $L=100$ and for a $2 \mathrm{~d}$ square lattice is $10 \times 10$ (or $20 \times 20$ in certain cases for checking the dependence on lattice size), with toroidal boundary conditions. From now on we fix the coupling constant to $J=1$ without loss of generality. We generated 2000 configurations for each $(T, H) ; 1000$ are used as training data for the machine, while the remaining 1000 compose the test data to avoid overlearning conditions of RBM. Here we note that nonlocal or cluster algorithms like the Swendsen-Wang algorithm could be also useful and applied for giving more accurate data on the critical region.

\section{B. RBM flow of configurations}

By the end of unsupervised learning of the RBM, the probability distributions of the training data $q\left(v_{i}\right)$ and the output reconstructed data $p\left(v_{i}\right)$ are not identical since the KL divergence $\operatorname{KL}(q \| p)$ practically does not become zero. To generate a flow of probability distributions we use as an input the reconstructed data into the $\mathrm{RBM}$, to obtain a new output with a probability distribution $\tilde{p}\left(v_{i}\right)$. Doing this procedure iteratively, we obtain a flow of probability distributions $q\left(v_{i}\right) \rightarrow p\left(v_{i}\right) \rightarrow$ $\tilde{p}\left(v_{i}\right) \rightarrow \cdots$.

When a probability distribution $p$ is given, we can obtain a concrete example of the spin configurations by replacing the expectation value $\left\langle v_{i}\right\rangle_{p}$ at each site with \pm 1 with the probability $\left(1 \pm\left\langle v_{i}\right\rangle_{p}\right) / 2$. Then the flow of probability distribution can be regarded as the flow of the spin configurations and it can be thought as an "RBM flow" of spin configurations as in [34]. The RBM learning and the process of reconstruction under the learned distributions to produce the RBM flow are schematically presented in Fig. 3.

\section{Neural network to measure $T$ and $H$}

By generating the RBM flow, we get new spin configurations with various probability distributions. In order to study them, we need to specify the temperature $T$ and external magnetic field $H$ of the reconstructed RBM spin configurations. This can be done in several ways; here we choose to do it by means of machine learning. We use a second independent neural network (NN) to perform a supervised learning of Ising configurations. This plays the role of an independent thermometer to identify the temperature of the RBM flow. For training the NN, we use the same training data for the RBM. Our NN has 100 neurons in the input layer $z_{i}^{(i)}$ and 200 neurons in the output layer $z_{\mu}^{(\text {o) }}$, since we have $20 \times 10$ combinations of $(T, H)$ in the training data. In addition, it is shallow and has only one hidden layer $z_{a}^{(\mathrm{h})}$ with 150 neurons. The activation function for the hidden layer and the output layer is chosen to be tanh:

$$
\begin{aligned}
& z_{a}^{(\mathrm{h})}=\tanh \left(\sum_{i} z_{i}^{(\mathrm{i})} W_{i a}^{(\mathrm{h})}+b_{a}^{(\mathrm{h})}\right), \\
& z_{\mu}^{(\mathrm{o})}=f\left(\tanh \left(\sum_{a} z_{a}^{(\mathrm{h})} W_{a \mu}^{(\mathrm{o})}+b_{\mu}^{(\mathrm{o})}\right)\right),
\end{aligned}
$$

where $W_{i a}^{(\mathrm{h})}, W_{a \mu}^{(\mathrm{o})}$ are the weight matrices, and $b_{a}^{(\mathrm{h})}, b_{\mu}^{(\mathrm{o})}$ are the biases. In the output layer, we use the softmax function $f(x)$ to get the probability distribution for $(T, H)$. The answer $(T, H)$ for each training data is given in the one-hot representation

$$
d_{\mu}=(0, \ldots, 0,1,0, \ldots, 0)=\delta_{\mu \nu},
$$

where we parametrize $v=2(T+20 H)$ so that the index accommodates all our parametrizations as $v=0,1, \ldots, 199$. Therefore, for training the $\mathrm{NN}$, we try to minimize the KL divergence of the answer $d_{\mu}$ and the output $z_{\mu}^{(\mathrm{o})}$ for the training data. Here we use the method of back propagation to optimize the weight matrices and biases. We set the learning rate 0.1 and the learning epoch 25000 . After training this NN, each of the output neurons $z_{\mu}^{(o)}$ shows the probability of each combination $(T, H)$ when a spin configuration is input into the input 


\section{Output reconstructed data}
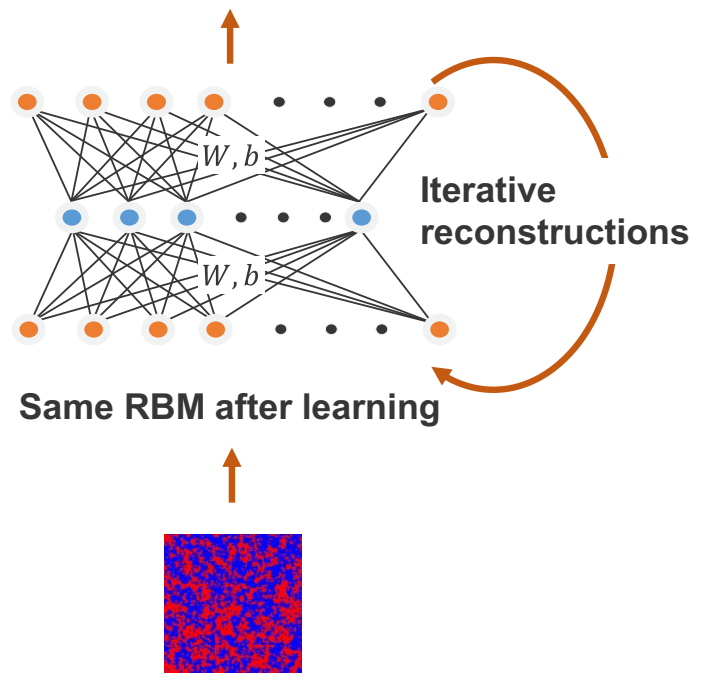

Input Monte-Carlo data

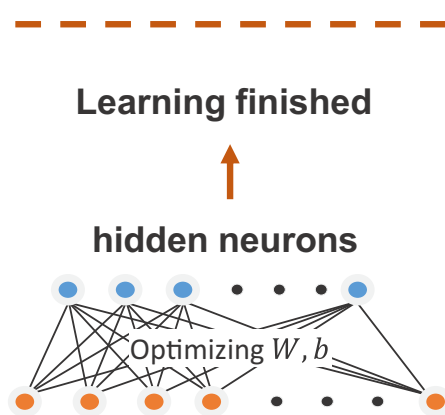

visible neurons

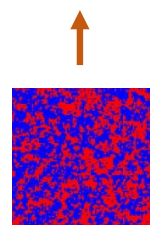

Input Monte-Carlo data

FIG. 3. During the learning of the RBM we optimize its parameters appropriately. Then the trained RBM is used to reconstruct data from the learned distribution. This data are used as an input to the same RBM machine to generate the flow through iterative reconstructions.

neurons $z_{i}^{(\mathrm{i})}$. Then we identify the measured $(T, H)$ by the NN as that with the highest probability: We test our NN on newly produced MC spin configurations to confirm the validity of the prediction of the right $(T, H)$.

\section{RBM FIXED POINTS AND ISING MODEL THERMODYNAMICS}

In this section we study the RBM flow using the NN to evaluate the probability distribution of spin configurations in the $(T, H)$ space. Along the RBM flow, i.e., the iterative reconstruction of configurations, the peaks of the probability

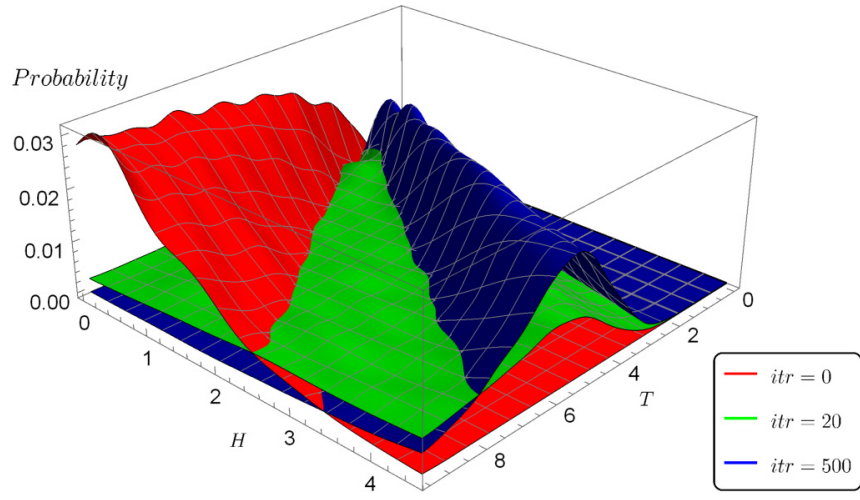

FIG. 4. RBM flow of configurations in 1d Ising model with number of hidden units $N_{h}=9$. Notice that after 20 iterative reconstruction of configurations a well-formed probability distribution already appears away from the initial configuration. After 500 iterations the flow has a sharp probability maximum. The dimensionful quantities are normalized with the constant coupling constant.

distributions behave according to a certain pattern and finally converge to special points of $(T, H)$, which we call the "RBM fixed points." In the special case that the external magnetic field is zero the RBM fixed point would match to the critical point of the theory. For fixed and nonzero magnetic field, we show that these points are identical to the ones that maximize the specific heat of the system suggesting an alternative way that the RBM recognizes the phase transitions. We work with the $1 \mathrm{~d}$ and $2 \mathrm{~d}$ Ising models, and we establish a correspondence between the thermodynamic properties of the Ising model and the RBM flows.

It turns out that the RBM flow depends heavily on the number of hidden neurons $N_{h}$ for both the Ising chain and lattice. We may classify the behaviors into two types, one type which is interesting for us occurs for a low number of hidden neurons, $N_{h}=9,16$, while the other type occurs for higher number $N_{h}=25, \ldots, 100$. In the former type, the RBM iterative reconstruction of configurations flows to fixed points at finite $(T, H)$, as shown for example in Fig. 4, while in the later type overlearning seems to occur.

Using a low number of neurons, it turns out that the behavior of the flow is not sensitive to the initial conditions of the physical system we use to generate it. The iterative reconstruction of configurations ends at the same fixed points irrespective of the starting configuration, hinting already at the capability of the RBM to extract certain physical properties of the system.

Below we perform the machine learning analysis and elaborate concretely on the points discussed above.

\section{A. RBM in 1d Ising model}

Keeping the hidden neurons $N_{h} \leqslant 16$ to avoid overlearning, we generate the RBM flow for the spin chain with the initial configuration at $(T, H)=(9.5,0.5)$, without loss of generality as already mentioned.

The generated RBM flow as a function of the magnetic field and temperature is depicted in Fig. 4. The probability distribution leads to a well-deformed peak after only about 20 iterative reconstructions, while for 500 iterations the peak 


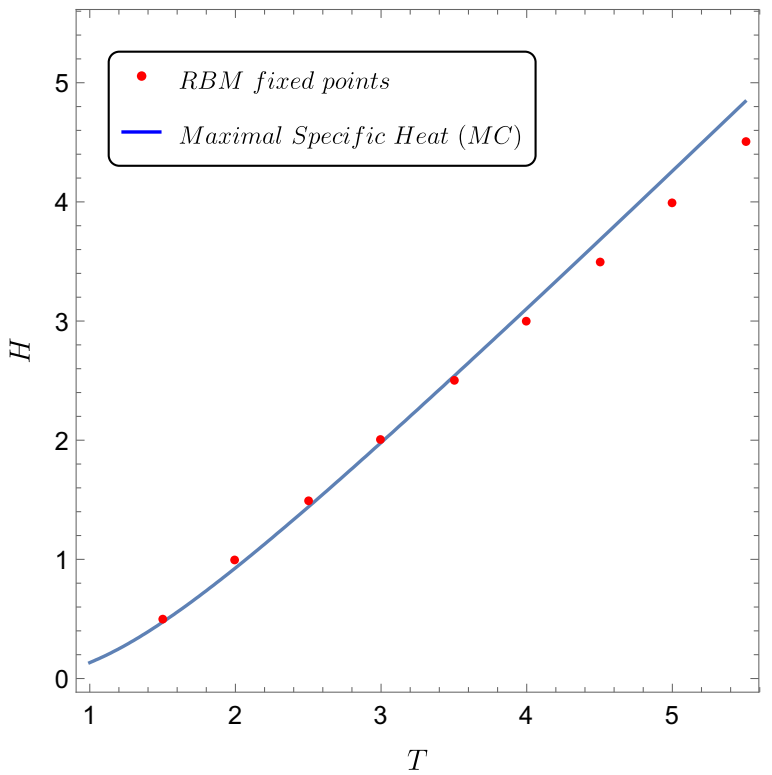

FIG. 5. The correlation between the RBM fixed points and the maximal points of specific heat for a fixed magnetic field, in the $1 \mathrm{~d}$ Ising model. The solid line is the numerical maximization of the analytic specific heat in the thermodynamic limit of a large lattice. It follows closely the RBM maximal probability in the parametric space. At low temperatures the RBM does produce clear maxima and is inconclusive.

of the flow becomes clearly identified. For nonzero magnetic field the RG flow is nontrivial as has been described in Sec. II, and there is a well-posed question as to which configuration the RBM-generated flow corresponds. We compare the RBM fixed points with the maximal specific heat of the Ising model and we find a striking agreement (Fig. 5). The RBM flows to the points that happen to resemble the Ising model's phase transition. For nonvanishing magnetic field where no critical point occurs, we find that the fixed points of the RG flow are not recognized as the RBM fixed points; instead the latter tend to recognize the ones with maximum specific heat. Our findings suggest that the machine learning generates flows with a tendency to approach certain configurations that resemble as closely as possible the ones of the phase transitions. A measure of this closeness to criticality is the maximization of the specific heat, which we find is in close agreement with the maximization of the probability of the reconstructed configurations.

The zero magnetic field RBM analysis at low temperature in spin chains would be also interesting since this is a special case, where the specific heat at the critical point $(T, H)=$ $(0,0)$ is finite. Nevertheless, the reconstructed flows in the near region do not seem to form clear probability peaks to extract conclusions.

The overlearned configuration occurs in the RBM with a larger number of hidden neurons $N_{h} \geqslant 25$, which flow to higher-temperature configurations. By increasing the number of neurons, the flow tends faster toward such configurations. The RBM has been overlearning about the configurations at higher temperature which are noiselike and we can check it by computing the eigenvectors of the weight matrix with large

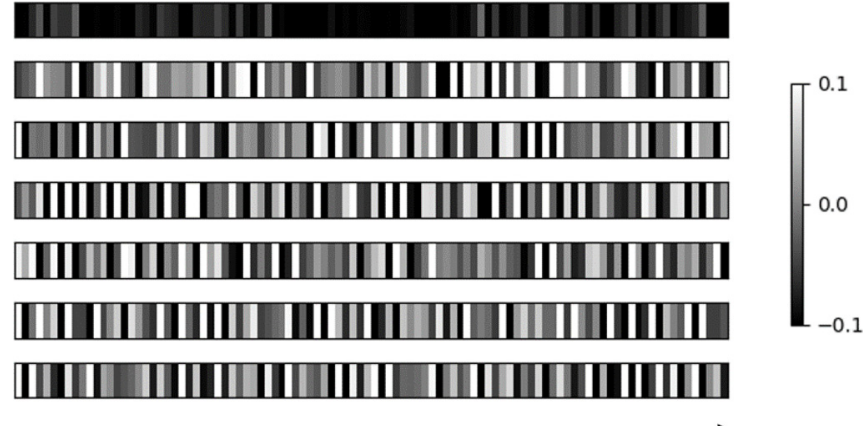

index $i$ of visible neurons

FIG. 6. The seven eigenvectors of the product of the weight matrix $\sum_{a} W_{i a} W_{j a}$ with the largest eigenvalues for 100 visible neurons. The uncorrelated distribution of eigenvector elements between the nearest neurons signals overlearning on noiselike configurations.

eigenvalues to show that their vast majority are noiselike, resulting in noiselike output data, Fig. 6. As an additional test to show such overlearning is not an artifact of the data or the RBM parameters used, we have also trained the RBM with $N_{h}=9$ and 16 using only high-temperature spin configurations, then confirmed that the RBM reconstructs the noisy high-temperature configurations as it learns.

\section{B. RBM in 2d Ising model}

The $2 \mathrm{~d}$ Ising model has a phase transition and an unstable critical point on the RG flow for $(T, H)=\left(T_{c}, 0\right)$ offering a richer phase diagram to study our claims for the RBM flow compared to that of the spin chain. We generate RBM flows using a low number of hidden neurons, starting from the configurations at $(T, H)=(9.5,1.0)$ without loss of generality. The three-dimensional plot of the flow as a function of the magnetic field and temperature is depicted in Fig. 7. The probability distribution leads to a well-deformed peak after 500 reconstructions. The RBM fixed points follow a curve on the $(T, H)$ plane that is defined as the maximum of the probability distribution.

In the absence of the external magnetic field, our RBM fixed point coincides with the Ising RG flow critical point as was observed in [34]. At the critical point the specific heat

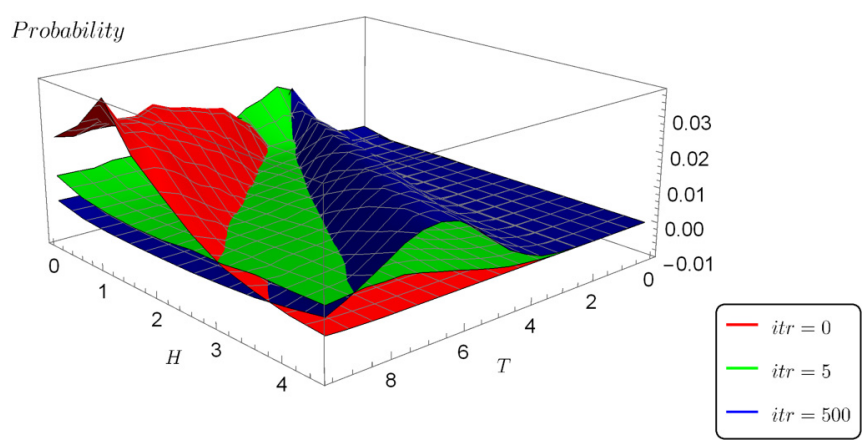

FIG. 7. RBM flow of configurations in the $2 \mathrm{~d}$ Ising model with number of hidden units $N_{h}=9$. The flow forms a pattern after a small number of iterations already and in 500 iterations it already has a clear peak. 


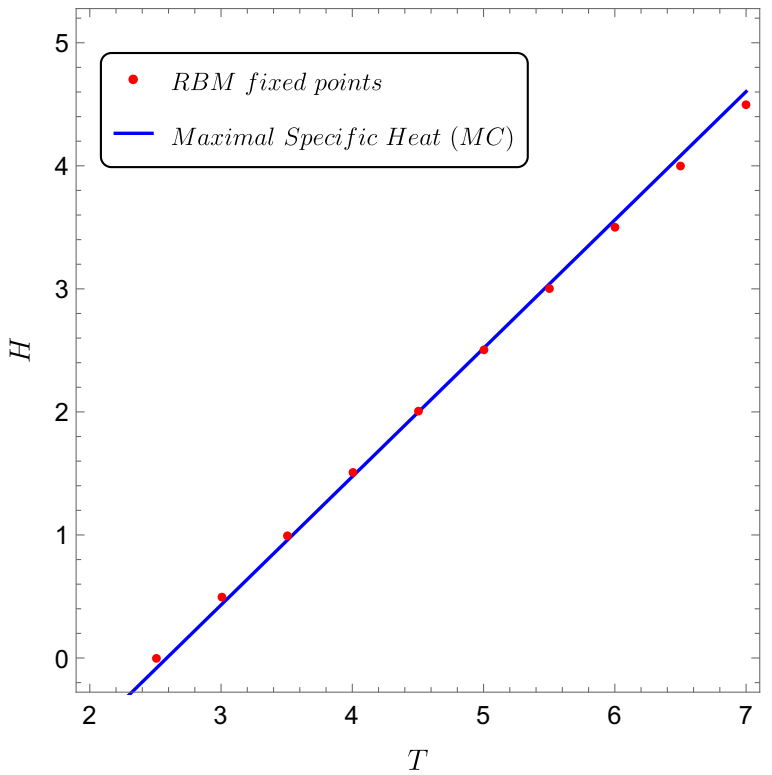

FIG. 8. RBM fixed points and maximal points of specific heat in the $2 \mathrm{~d}$ Ising model for reconstructed flows with fixed magnetic fields.

has a maximum and diverges, and therefore it is one of the points that maximize the specific heat for given $H=0$. By applying a fixed external magnetic field the Ising model does not undergo a phase transition, although the RG flow has fixed points. We observe that our RBM flow of iterative reconstruction of configurations does not end up at the fixed points of the RG Ising flow; instead it tends to points of finite $(T, H)$, which correspond to configurations that resemble the closest possible ones to the phase transition. For any combination of $(T, H)$ with fixed magnetic field the RBM flow tends toward the points that correspond in the Ising model to the maximum of the specific heat (Fig. 8). The RG critical point at zero magnetic field happens to be one of the points that satisfies the maximum specific heat criterion.

We note that for RBMs with a higher number of hidden neurons, $N_{h} \geqslant 25$, the noise takes over and the flow approaches higher temperatures, while as $N_{h}$ increases the flow goes to these points more rapidly. The overlearning in presence of external field occurs for a lower number of hidden neurons than without it, which has been found to occur for $N_{h}>N_{v}=100$ [34]. To obtain an analytic prediction of the ideal number of hidden nodes for the best optimized learning is an open and interesting question. The numerical pattern we find here can be important input in developing a more analytic relation between the RBM flow and the RG flow, a very challenging task.

\section{Ising models and RBM flows}

The question that arises is what is the feature of the Ising model that signals to the machine learning to flow to certain configurations. For nonvanishing magnetic field the RBM flows to configurations that have certain thermodynamic properties. In particular these maximize the specific heat for fixed magnetic field. Our analysis suggests that the RBM fixed points are the ones that correspond to configurations that maximize certain thermodynamic properties, and resemble closer the state of the phase transition of the physical system even when the physical system does not undergo such transition. When the system undergoes a phase transition, the RBM identifies the critical fixed point by the same criterion since at criticality the specific heat diverges. We highlight at this point that we provide no prior knowledge to the RBM about the criticality of the physical system and its Hamiltonian, to guarantee that the RBM flow extracts the features of input data and recognizes the relevant and irrelevant degrees of freedom spontaneously.

Our result implies the possibility of an effective mapping between the way that the machine learns and the thermodynamics of the Ising model. If this is realized, a large number of applications would follow, both in the deeper understanding of the way that the machine learning performs, given that the Ising model is very well understood, and in extending the applications of ML to physical models. Recognizing complex holographic phase transitions, like the ones of [40], by ML and extracting the relevant physical information would be such a direction.

\section{CRITICAL EXPONENTS WITH MACHINE LEARNING}

We have started by assuming no knowledge of an existing phase transition, and have employed unsupervised learning to obtain the critical point in the Ising model at zero magnetic field. It is known that the accuracy of the training on learning the two-dimensional properties of the Ising model depends mainly on the number of neurons in the first hidden layer of the network and not on other model details such as the model type and the network depth [41]. Taking into account that shallow networks are very efficient at representing physical probability distributions associated with Ising systems near criticality we demonstrate here how to compute the critical exponents of the model based exclusively on the machine learning.

The principles of scaling and universality play important roles in the theory of phase transitions and critical phenomena. Observable quantities exhibit power law singularities in the variable $\delta T=T-T_{c}$ in the vicinity of the critical temperature, where the proportionality coefficients are functions of dimensionless combinations of the dimensionful Ising model parameters. In this vicinity the scaling and universality hypotheses predict that the leading singular part of the free energy can be expressed through a universal function for all systems in a given universality class.

Here we demonstrate how to compute the critical exponent associated with the magnetization using the machine learning. The magnetization (8) can be expanded around the critical point to give

$$
m \sim 1.222{\frac{\left|T_{c}-T\right|^{\frac{1}{8}}}{T_{c}}} .
$$

The order parameter critical exponent is $\beta=1 / 8$, which is independent of the lattice type considered.

We take advantage of the fact that the RBM flow recognizes and approaches $T_{c}$, and that we get the vast majority of new configurations at around $T_{c}$ which are reconstructed by 


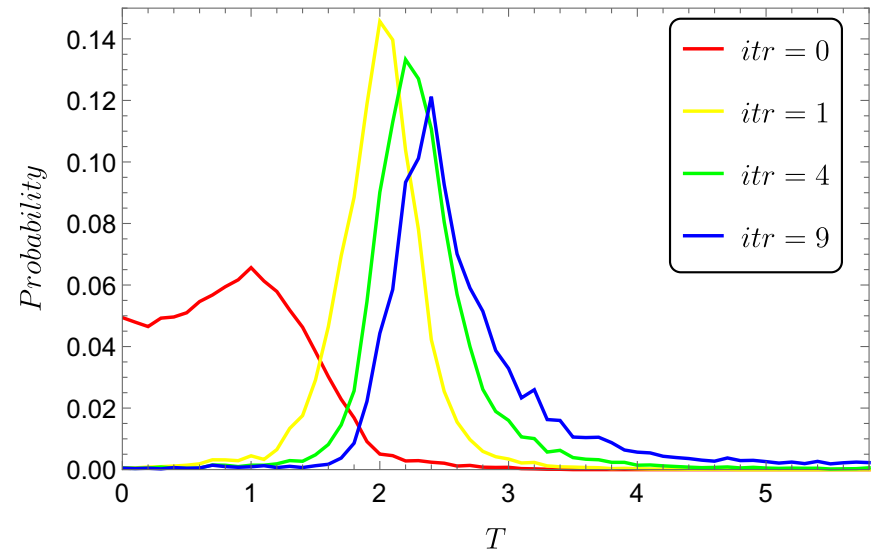

FIG. 9. RBM flow of configurations at $H=0$ in the $2 \mathrm{~d}$ Ising model (when $N_{h}=81$ ) with a variety in the number of iterative reconstructions. The RBM fixed point located at the peak of the line corresponds to the largest number of iterations and is at $T \simeq 2.4$. The RBM starts from the configurations at $T=1$ as depicted in the zero-iteration line of the plot.

the RBM. In order to have large number of such configurations, we need to set $N_{h} \lesssim N_{v}$ so that the RBM flow arrives at $T_{c}$ after many iterations of reconstructions [42]. Then we use the RBM with $N_{h}=81$, which learns the configurations at various temperatures $T=0,0.1, \ldots, 5.9$ in the absence of magnetic field. For training and testing of the RBM, we prepare $1000+1000$ spin configurations at each temperature. Using the same configurations, we also train and test the $\mathrm{NN}$ to measure their temperature. This $\mathrm{NN}$ has 100,80 , and 60 neurons in the input, hidden, and output layers, respectively. The way of training of the RBM and the NN, including the learning rate and the learning epoch, is the same as in previous sections. Using these settings where we generate the flow, a representative example of the probability is presented in Fig. 9. Here we use the configurations at $T=0,0.1, \ldots, 1.9$ as the starting points. Originally we have the $2000 \times 20$ spin configurations at these temperatures and we make the RBM to iteratively reconstruct the configurations nine times. After that, we have the $4 \times 10^{5}$ configurations in total and many of them are at around $T_{c}$. Their temperatures can be measured using the $\mathrm{NN}$, where the $\mathrm{NN}$ outputs the probability distribution of the temperature, so we can regard the temperature with the highest probability as the estimated temperature.

To compute the magnetization we use the RBM configurations around $T_{c}$. For the configurations at each estimated temperature, we calculate the averaged absolute values of magnetization per site $\langle m\rangle$; a sample of the results is presented in Fig. 10. It is a relatively straightforward fitting problem, taking advantage of the fact that the RBM flow has a stable point that matches the Ising critical one, so the reconstructed Ising data would correspond to configurations around the critical temperature. As an extra feature on the figure, we show the Bethe-Peierls approximation since we notice that it agrees well with the RBM reconstructed spin configurations. Possibly this is due to the approximations taking place while we are computing the RBM partition function (e.g., the CD method) which resemble the mean field approximation.

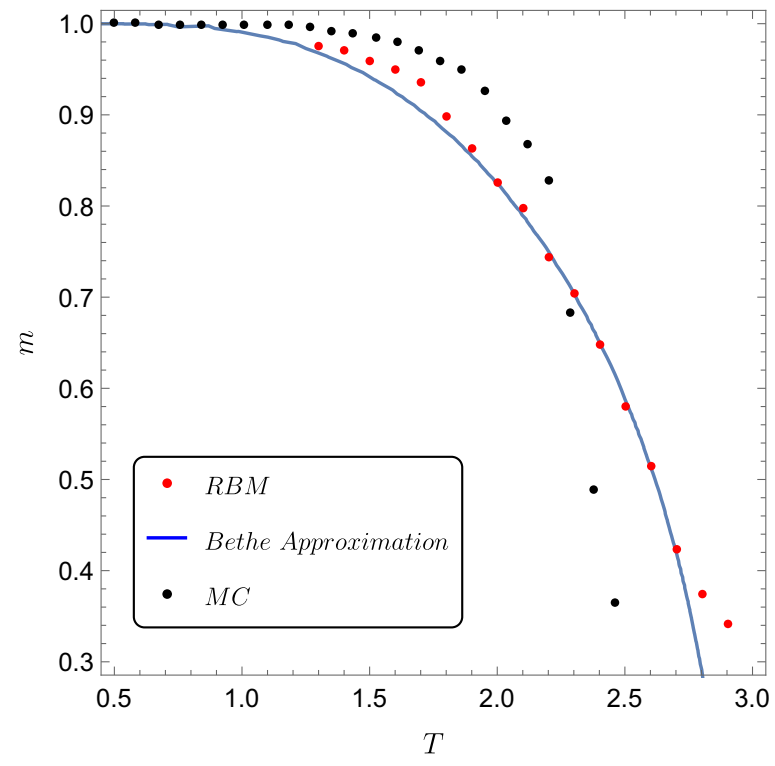

FIG. 10. Magnetization versus temperature. The RBM, MC simulations, and Bethe-Peierls approximation are relatively close. We estimate the critical exponent near criticality by using the RBM configurations.

By logarithmical fitting we estimate the critical exponent using the RBM configurations around the critical temperature that the RBM provides to obtain

$$
\langle m\rangle \sim 0.931\left|T-T_{c}\right|^{0.127},
$$

which gives $\beta \simeq 0.127$, close to the actual value $\beta=0.125$. Our fitting has statistical errors that depend on the quantity of the data we collect, and in practice can be reduced, since here we have used a wider range of values spanning $T-T_{c} \sim 0.4$. We demonstrate successfully how to compute thermodynamic quantities on the samples generated by the Boltzmann machines and show that they faithfully reproduce those calculated directly from the Monte Carlo samples. We point out that we have not given in advance any information to the RBM on where the phase transition occurs and have assumed no knowledge of the Hamiltonian of the model.

Our results clearly demonstrate that RBMs with standard Monte Carlo methods can be used as a powerful tool to study physical models. We have shown that the performance of the $\mathrm{RBM}$ in the reconstruction of the thermodynamic quantities and the computation of the critical exponents are in direct comparison of the relevant results produced by the theoretical models and MC methods.

\section{CONCLUSION AND DISCUSSION}

In this work we have trained an RBM to produce a stochastic model of a thermodynamic probability distribution. The generated RBM flow, i.e., the iterative reconstruction of data, is produced by configurations of the one-dimensional and two-dimensional Ising models in the presence of external magnetic field. The RBM has only the information of the binary images corresponding to spin microstates produced by the MC, and has no direct prior knowledge about the physical properties of the system, the existence of the criticality, and 
its Hamiltonian. We find that the flow of the RBM reconstruction data approaches the spin configurations of the maximal possible specific heat which resembles, or matches in the case of zero external field, the near-criticality region of the Ising model. In the special case of the vanishing magnetic field, the RBM flow converges to the critical point of the renormalization group flow of the lattice model. Our work confirms that the RBM learns the probability distribution that corresponds to the critical phase transition. Our results suggest an alternative explanation of how the machine identifies the physical phase transitions, by recognizing certain properties of the configuration like the maximization of the specific heat, or the maximization of the correlation length, instead of associating directly the recognition procedure with the RG flow and its fixed points.

Motivated by our results it is intriguing to ask if a map between the thermodynamic quantities or correlators in lattice models, with certain quantities for the RBM, can be established, where a reasonable concept of equilibrium in the RBM should be also defined. If this is realized, a deeper understanding of the way that the machine learning performs will be achieved and the ML method will find application to a larger variety of physical models.

Having studied the properties of the RBM flows, we have then demonstrated the computation of the thermodynamic quantities on the samples generated by the Boltzmann machines, to show that they potentially reproduce those calculated directly from the Monte Carlo samples. By looking at the zero magnetic field model and without giving information to the machine about the criticality of the system and its Hamiltonian, we compute the critical exponent associated with magnetization from the reconstructed data to find satisfactory agreement. This is not surprising; once we accept the fact that the RBM flow has a stable point that matches the Ising critical one, the reconstructed Ising data correspond to configurations around the critical temperature. Then the computations of the critical exponents is a straightforward fitting problem on the reconstructed data. Our findings demonstrate that RBMs with standard Monte Carlo methods can be used as a powerful tool to study physical models and to reconstruct the thermodynamic quantities accurately.

\section{ACKNOWLEDGMENTS}

The authors acknowledge useful conversations with Konstantinos Anagnostopoulos, Chong-Sun Chu, Robert de Mello Koch, Satoshi Iso, Jonathan Miller, and Reuven Pnini. S.S.F. is partially supported by a Grant-in-Aid for Scientific Research (No. 16K17711) from the Japan Society for the Promotion of Science (JSPS). D.G. is supported by the Ministry of Science and Technology of Taiwan (MOST) under the grant 104-2112-M-007-001 -MY3.
[1] G. E. Hinton, Trends Cognit. Sci. 11, 428 (2007).

[2] Y. LeCun, Y. Bengio, and G. Hinton, Nature (London) 521, 436 (2015).

[3] L. Wang, Phys. Rev. B 94, 195105 (2016).

[4] G. Torlai and R. G. Melko, Phys. Rev. B 94, 165134 (2016).

[5] S. J. Wetzel, Phys. Rev. E 96, 022140 (2017).

[6] W. Hu, R. R. P. Singh, and R. T. Scalettar, Phys. Rev. E 95, 062122 (2017).

[7] J. Carrasquilla and R. G. Melko, Nat. Phys. 13, 431 (2017).

[8] A. Tanaka and A. Tomiya, J. Phys. Soc. Jpn. 86, 063001 (2017).

[9] S. J. Wetzel and M. Scherzer, Phys. Rev. B 96, 184410 (2017).

[10] L.-F. Arsenault, O. Anatole von Lilienfeld, and A. J. Millis, arXiv:1506.08858.

[11] G. Carleo and M. Troyer, Science 355, 602 (2017).

[12] M. R. Hush, Science 355, 580 (2017).

[13] Z. Cai and J. Liu, Phys. Rev. B 97, 035116 (2018).

[14] G. Torlai, G. Mazzola, J. Carrasquilla, M. Troyer, R. Melko, and G. Carleo, Nat. Phys. 14, 447 (2018).

[15] P. Baldi, P. Sadowski, and D. Whiteson, Nat. Commun. 5, 4308 (2014).

[16] S. S. Schoenholz, E. D. Cubuk, D. M. Sussman, E. Kaxiras, and A. J. Liu, Nat. Phys. 12, 469 (2016).

[17] P. Broecker, J. Carrasquilla, R. G. Melko, and S. Trebst, Sci. Rep. 7, 8823 (2017).

[18] Y. Zhang, R. G. Melko, and E.-A. Kim, Phys. Rev. B 96, 245119 (2017).

[19] F. Schindler, N. Regnault, and T. Neupert, Phys. Rev. B 95, 245134 (2017).
[20] Y. Zhang and E.-A. Kim, Phys. Rev. Lett. 118, 216401 (2017).

[21] E. P. L. van Nieuwenburg, Y.-H. Liu, and S. D. Huber, Nat. Phys. 13, 435 (2017).

[22] E. van Nieuwenburg, E. Bairey, and G. Refael, Phys. Rev. B 98, 060301(R) (2018).

[23] P. Suchsland and S. Wessel, Phys. Rev. B 97, 174435 (2018).

[24] K. Hashimoto, S. Sugishita, A. Tanaka, and A. Tomiya, Phys. Rev. D 98, 046019 (2018).

[25] K. Hashimoto, S. Sugishita, A. Tanaka, and A. Tomiya, Phys. Rev. D 98, 106014 (2018).

[26] P. Mehta, M. Bukov, C.-H. Wang, A. G. R. Day, C. Richardson, C. K. Fisher, and D. J. Schwab, Phys. Rep. 810, 1 (2019).

[27] C. Bény, arXiv:1301.3124.

[28] P. Mehta and D. J. Schwab, arXiv:1410.3831.

[29] A. Paul and S. Venkatasubramanian, arXiv:1412.6621.

[30] K.-I. Aoki and T. Kobayashi, Mod. Phys. Lett. B 30, 1650401 (2016).

[31] H. W. Lin, M. Tegmark, and D. Rolnick, J. Stat. Phys. 168, 1223 (2017).

[32] S. Foreman, J. Giedt, Y. Meurice, and J. Unmuth-Yockey, EPJ Web Conf. 175, 11025 (2018).

[33] M. Koch-Janusz and Z. Ringel, Nat. Phys. 14, 578 (2018).

[34] S. Iso, S. Shiba, and S. Yokoo, Phys. Rev. E 97, 053304 (2018).

[35] G. E. Hinton, in Neural Networks: Tricks of the Trade, Lecture Notes in Computer Science, edited by G. Montavon, G. B. Orr, and K.-R. Müller (Springer, Berlin, 2012), p. 599.

[36] L. Onsager, Phys. Rev. 65, 117 (1944). 
[37] T. D. Lee and C. N. Yang, Phys. Rev. 87, 410 (1952).

[38] G. E. Hinton, Neural Comput. 14, 1771 (2002).

[39] T. Tieleman, in Proceedings of the 25th International Conference on Machine Learning, ICML '08 (ACM, New York, 2008), pp. 1064-1071.

[40] D. Giataganas, U. Gürsoy, and J. F. Pedraza, Phys. Rev. Lett. 121, 121601 (2018).
[41] A. Morningstar and R. G. Melko, J. Mach. Learn. Res. 18, 1 (2017).

[42] In [4] it has already been noticed that the number of hidden nodes required to reproduce faithfully the MC results had to be increased around criticality to reconstruct more faithfully certain thermodynamic quantities. This reflects the increase of the fluctuations near the critical region. 